\title{
Evaluating the accessibility to healthcare facilities under the Chinese hierarchical diagnosis and treatment system
}

\author{
Jingkai Yue, ${ }^{1}$ Qiang Fu, ${ }^{2}$ Ying Zhou, ${ }^{3}$ Yue Zhang, ${ }^{4}$ Jie Ning, ${ }^{5}$ Gang Yin, ${ }^{6}$ Hongbing Tao ${ }^{6}$ \\ ${ }^{1}$ Logistics Department, The Central Hospital of Wuhan, Tongji Medical College, Huazhong University of \\ Science and Technology, Wuhan, China; ${ }^{2}$ Department of Community Health, School of Arts and Sciences, \\ Tufts University, Medford, MA, USA; ${ }^{3}$ Human Resource Department, Wuhan Forth Hospital, Puai Hospital, \\ Tongji Medical College, Huazhong University of Science and Technology, Wuhan, China; ${ }^{4}$ Department of \\ Public Administration, Wuhan University of Science and Technology, Wuhan, China; ${ }^{5}$ Medical Insurance \\ Office, Sun Yat-sen University Cancer Center, State Key Laboratory of Oncology in South China, \\ Collaborative Innovation Center for Cancer Medicine, Guangzhou, China; ${ }^{6}$ Department of Health \\ Administration, School of Medicine and Health Management, Tongji Medical College, Huazhong University \\ of Science and Technology, Wuhan, China
}

\begin{abstract}
An accurate assessment of current healthcare resource allocations is essential to address existing inequities in the hierarchical diagnosis and treatment system introduced in China. The data
\end{abstract}

\footnotetext{
Correspondence: Hongbing Tao, Department of Health Administration, School of Medicine and Health Management, Tongji Medical College, Huazhong University of Science and Technology, Wuhan, China.

Tel.: +86.0278369253. E-mail: hhbtao@hust.edu.cn
}

Key words: Spatial accessibility; healthcare facilities; hierarchical diagnosis and treatment system; China.

Acknowledgements: the authors sincerely thank the Zhongshan Health Bureau for providing us with the data used in this study. The author also thanks Yuting Liao for her polishing work in the article.

Funding: this work was funded by the National Natural Science Foundation of China (Grant Number: 71774061).

Contributions: JY, HT, conceptualization; JY, methodology, formal analysis, original draft preparation; $\mathrm{YZ}$, software, investigation, visualization; QF, JN, YZ, validation; GY, resources; JN, data curation; HT, $\mathrm{QF}$, review and editing, supervision; HT, project administration, funding acquisition. All authors have read and agreed to the published version of the manuscript.

Conflict of interests: the authors declare no conflict of interests.

Received for publication: 6 March 2021

Revision received: 3 May 2021.

Accepted for publication: 4 May 2021.

(C) Copyright: the Author(s), 2021

Licensee PAGEPress, Italy

Geospatial Health 2021; 16:995

doi:10.4081/gh.2021.995

This article is distributed under the terms of the Creative Commons Attribution Noncommercial License (CC BY-NC 4.0) which permits any noncommercial use, distribution, and reproduction in any medium, provided the original author(s) and source are credited. come from statistical reports of local governments and the developer platform of Amap, a Chinese mobile map. The data were analysed using the hierarchical two-step floating catchment area method. By spatial accessibility analysis, the distribution of accessibility to hierarchical healthcare facilities in Zhongshan City, Guangdong Province was found to be uneven, with clustered high accessibility in the central, north-western and southern parts of the city. To enhance the capacity of healthcare services, the government should allocate healthcare resources rationally to better associate with population densities.

\section{Introduction}

In an effort to provide affordable and accessible medical services to all citizens, the 2005 World Health Assembly of the World health Organization (WHO) called on its Member States to achieve universal health coverage (UHC) (WHO, 2005). For a long time, China's health system has been faced with a range of inequalities resulting in fragmentation of services and low accessibility, expressed by people as ' $h$ ard and expensive to see a doctor' (Tediosi et al., 2016; Li and Fu, 2017; Liu et al., 2017; Shan et al., 2017). Equity in access to healthcare is one of the fundamental goals of realizing UHC (Shan et al., 2017) and in response to the appeal of the World Health Assembly, the Chinese Government deployed a series of reform measures and launched an ambitious plan of healthcare system reform in April 2009, with the goal of providing universal coverage of essential health services for all Chinese citizens by 2020 ( $\mathrm{Li}$ and $\mathrm{Fu}$, 2017). In 2015, the General Office of the State Council issued 'Guiding Opinions on Promoting the Construction of a Hierarchical Diagnosis and Treatment System' aimed to solve some long-standing problems, such as the imperfect layout of the medical service system, the insufficiency in high-quality healthcare resources and the unreasonable allocation of healthcare resources.

The medical service mainly classifies patients according to the severity of disease and difficulty of treatment. Different levels of healthcare institutions are responsible for the treatment of various diseases, and referrals between primary care and secondary (or tertiary) hospitals are encouraged to improve the efficiency of the system and reduce healthcare expenses (Qingyue et al., 2019). To achieve this goal, the Chinese Government is optimizing the distribution of healthcare resources by clarifying the functions at dif- 
ferent levels and promoting resource sharing. In recent years, remarkable results were achieved in the hierarchical diagnosis and treatment system, with the basic conditions of primary healthcare institutions continuously ameliorated and the service capacity significantly strengthened (Li et al., 2017; Yip et al., 2019). According to the National Health Commission (2019). The number of primary healthcare facilities rose from 882,153 in 2009 to 954,390 in 2019 , an increase of $8.2 \%$; while that of health technicians went up by $59.6 \%$ (from 1.83 to 2.92 million). Simultaneously, the number of beds increased by $48.3 \%$ (1.10 to 1.63 million), while there were $33.6 \%$ more primary healthcare visits (from 3.39 to 4.53 billion) and the number of patient transferrals from higher-level hospitals to lower-level hospitals also grew. These figures demonstrate that China's hierarchical diagnosis and treatment system is gradually improving (Liu et al., 2017; Tediosi et al., 2016). Although the new system seems to have yielded impressive results at the primary level, some problems that have long haunted China's healthcare system still persist. For example, an uneven distribution of healthcare resources, inequity between urban and rural areas, low accessibility to healthcare services and the monopoly benefit by large hospitals. A study by Yue et al. (2018) shows that intra-regional differences were the main reason for the unbalanced allocation of healthcare resources in mainland China, while Jay and David (2016) found that the distribution of hospital beds was highly concentrated spatially based on data from 2043 counties nationwide, with the most inequitable distribution of resources in the western provinces. There was also a significant positive correlation between fiscal revenue and the density of hospital beds in each county. In addition, official statistics showed that China's tertiary-level hospitals had $52.1 \%$ of the physicians, $65.1 \%$ of the nurses and $45.6 \%$ of the beds, while the number of consultations continued to rise on an annual basis (National Health Commission, 2020). China is still confronted with many chal- lenges to fully establish a systematic and well-connected medical system (Li et al., 2017; Song et al., 2018). As people's need for healthcare grows, reasonable allocation of public healthcare facilities, an important issue for basic public service facilities and a long-standing public concern, comes under the spotlight. An accurate assessment of the current healthcare resource allocations is essential to understand and address existing inequities needed to narrow the gaps in healthcare access and promoting overall population health (Wang et al., 2018).

With the rapid development of geographic information systems (GIS), accessibility can be straightforwardly estimated and thus become an indicator of the ease and quantity of access to public services in different locations (Banister, 1996; Walter and Hansen, 2007). Based on such measurements, we can identify areas or populations that face a shortage of opportunities and access to existing resources (Tao et al., 2020). Methods for measuring spatial accessibility can broadly be divided into four categories: the provider-to-population ratio; the travel impedance to the nearest provider; the average travel impedance to any provider; and the gravity models (Guagliardo, 2004) (Table 1). The two-step floating catchment Area (2SFCA) method (Radke and Lan, 2000; Luo and Qi, 2009) was developed from the gravity model and has been favoured. Due to further improvement and application of this basic formula, numerous scholars have carried out abundant research on the accessibility to public service facilities using this approach (Tao and Cheng, 2016) (Figure 1).

Spatial accessibility is an indicator of potential healthcare provision and considered an important component in assessing overall population access to healthcare. For example, Bauer et al. (2017) focused on analyzing accessibility to general and specialized obstetric units in England and Germany with regard to urbanity, area deprivation and neonatal outcome using routine data. Pan et al. (2015) compared the results from the conventional measure-

Table 1. Approaches to spatial accessibility.

\begin{tabular}{|c|c|c|}
\hline Method & Definition & Advantage - Disadvantage \\
\hline Provider-to-population ratios & $\begin{array}{l}\text { Also known as the supply-to-demand ratio; } \\
\text { refers to the ratio of supply to demand in } \\
\text { a bounded region and is generally the ratio } \\
\text { of the number of hospitals, primary healthcare } \\
\text { facilities, physicians and beds to the population } \\
\text { in the region; commonly used indicators include: } \\
\text { physicians per } 1000 \text { population, beds per } \\
1000 \text { population, nurses per } 1000 \text { population, etc. }\end{array}$ & $\begin{array}{l}\text { Easy to understand and easy to calculate; can be calculated } \\
\text { without GIS tools } \\
\text { Results are influenced by administrative boundaries, } \\
\text { i.e. they do not account for the accessibility of } \\
\text { demand points near administrative area boundaries } \\
\text { Lacks consideration of travel time and distance }\end{array}$ \\
\hline $\begin{array}{l}\text { Travel impedance to the } \\
\text { nearest provider }\end{array}$ & $\begin{array}{l}\text { Cost in time or distance for residents to access } \\
\text { healthcare services; mainly estimated by measuring } \\
\text { the travel impedance from the point of demand } \\
\text { or the geometric centre of the patient's residence } \\
\text { to the health care facility by applying Euclidean } \\
\text { (straight-line) distances or a transportation network }\end{array}$ & $\begin{array}{l}\text { Easy to understand and calculate; assumes that people will } \\
\text { always choose the nearest provider, which is not entirely realistic } \\
\text { Ignores the supply-side situation and lacks } \\
\text { consideration of waiting time and busyness }\end{array}$ \\
\hline Average travel impedance to any provider & $\begin{array}{l}\text { Summation and average of the travel impedance from } \\
\text { all demand points or patient residences to all supply } \\
\text { points in the region }\end{array}$ & $\begin{array}{l}\text { Is a combined measure of accessibility and availability; } \\
\text { over-weighs the influence of providers located near the } \\
\text { periphery of the study area } \\
\text { Ignores the problem of patients crossing administrative } \\
\text { boundaries to seek care }\end{array}$ \\
\hline Gravity models & $\begin{array}{l}\text { A modified version of Newton's Law of Gravitation. } \\
\text { The larger the scale of the forces between the supply } \\
\text { and demand points, the larger the scale of the } \\
\text { measured service facilities, and the closer the } \\
\text { distance, the higher the level of accessibility }\end{array}$ & $\begin{array}{l}\text { Is a combined measure of accessibility and availability } \\
\text { without need to set a threshold range in advance; avoids } \\
\text { differences in results due to the choice of threshold range } \\
\text { Accessibility at the individual household level can be demonstrated; } \\
\text { determination of the } \beta \text {-value is subjective } \\
\text { Formulas too abstract for understanding and programming }\end{array}$ \\
\hline
\end{tabular}


ment approach with those by the 2SFCA approach, finding that the accessibility of catchment area varies significantly among regions in Sichuan Province. Indeed, the healthcare system is a complex hierarchical structure where facilities at different levels are distinct in size, function, quality of service and service area (Jin et al., 2019), characteristics that should be considered when measuring facility accessibility. Researchers have made great efforts in the study of the location-allocation problem of hierarchical facilities in recent decades. For example, Jin et al. (2019) proposed a hierarchical two-step floating catchment area (H2SFCA) method for measuring spatial accessibility to public healthcare resources by considering different levels of healthcare resource factors. Tao et al. (2020) further improved the method on this basis.

Current research on the accessibility to hierarchical healthcare facilities in China primarily focuses on extremely large cities, such as Beijing, Shanghai and Shenzhen ( $\mathrm{Gu}$ et al., 2019; Lu et al., 2019). Due to their economic power and political status, these cities have long held a monopoly on high-quality healthcare resources. However, as China's urbanization and reform of hierarchical diagnosis and treatment system make headway, the accessibility to hierarchical healthcare facilities in medium-sized cities also deserves attention. According to the Chinese Government's development plans, these cities will be the drivers of future urbanization (Central Committee of the Communist Party of China, 2020; Zhai and Hou, 2020). Still, only scant attention has been paid to the accessibility to healthcare facilities in medium-size cities, while the opening and use of application programming interfaces (APIs) provide new ideas for measuring journey times and distances in these cities, which partly compensate for the lack of timely data on the road network in medium-size cities (Song et al., 2013; Wang and $\mathrm{Xu}, 2011$ ). Since the reform and opening-up of China, a large number of highly urbanized areas have emerged, and our aim was therefore to measure the spatial accessibility to different levels of facilities under the hierarchical diagnosis and treatment system as this would provide guidance for the improvement of the current healthcare system and the spatial allocation of resources.

\section{Materials and methods}

\section{Study area}

The city of Zhongshan, a mid-sized city with a high level of urbanization, was chosen as study area because of its rapid economic and social development. It is located in the south-central part of Guangdong Province in the geographical centre on the western coast of the Guangdong-Hong Kong-Macao Greater Bay Area, which is currently vigorously promoted. Zhongshan has evolved into a medium-sized city with a resident population of 3.31 million and an administrative area of $1783.67 \mathrm{~km}^{2}$. By the end of 2018, its gross regional product reached 363.27 billion RMB (52.76 billion USD) resulting in an annual per capita disposable income of 48,804 RMB (7,088 USD) and 32,263 RMB (4685 USD) for urban residents and permanent rural residents, respectively - both higher than the national average (Zhongshan Bureau of Statistics, 2019). In addition, the city has adopted a point-based payment system on the basis of the diagnosis related groups (DRG) system, which is in line with the current payment method reform in China. Unlike other prefecture-level cities in China, Zhongshan is one of four such cities in China without a countylevel administrative hierarchy.

The traditional main urban area of Zhongshan includes subdistricts (township-level administrative divisions) such as $\mathrm{Xi} \mathrm{qu}$, Shiqi qu and Dong qu, which have high population densities. However, due to its well-developed township economy, the townships of Nantou, Dongfeng, Xiaolan and Guzhen in the Northeast and Sanxiang in the South also have large populations (Figure 2). The road network of Zhongshan City is densest in the main urban area and the north-western region (Figure 3). The demand for healthcare services in Zhongshan is on the rise, the number of outpatients in the city reached $36,365,900$ and the number of inpatients reached 585,700 in 2018 (Health Bureau of Zhongshan, 2018).

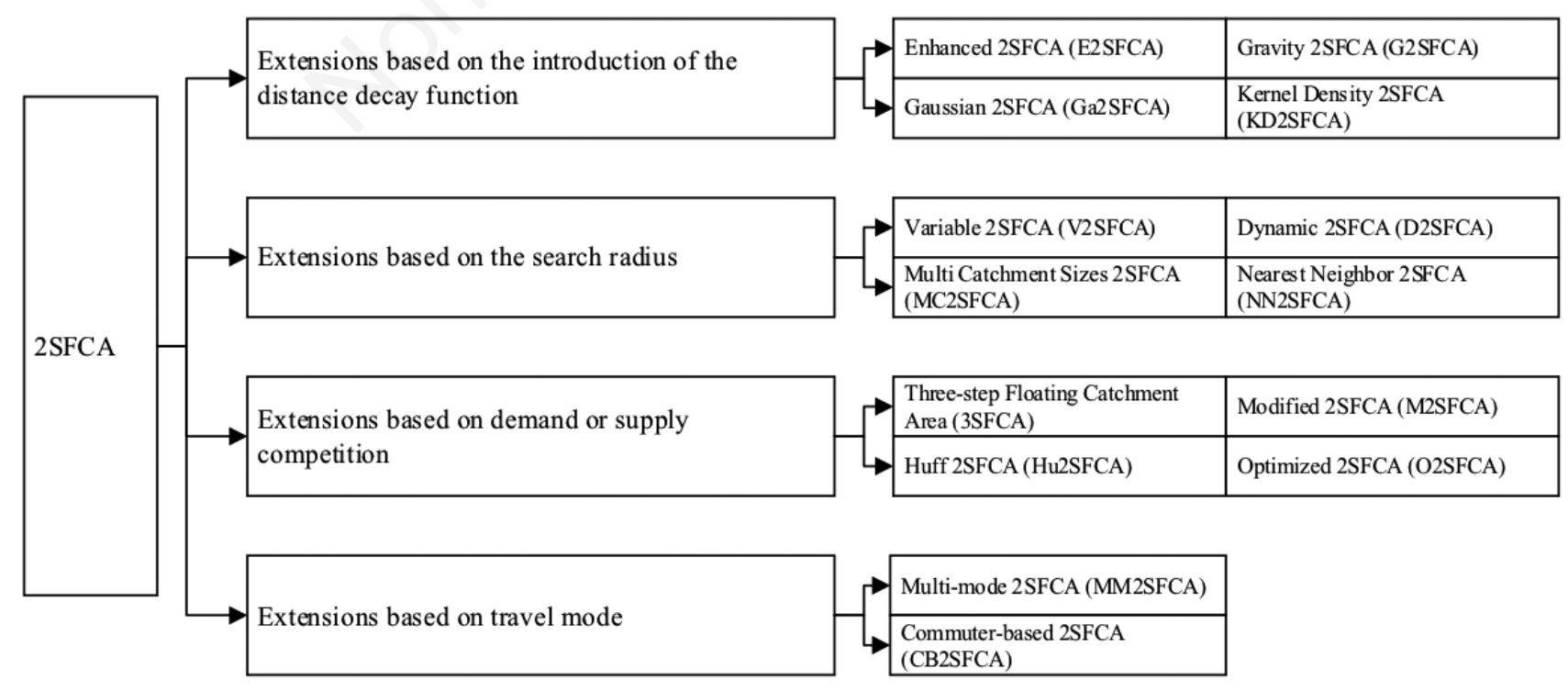

Figure 1. Diagrammatic presentation of the two-step floating catchment area (2SFCA) method. 


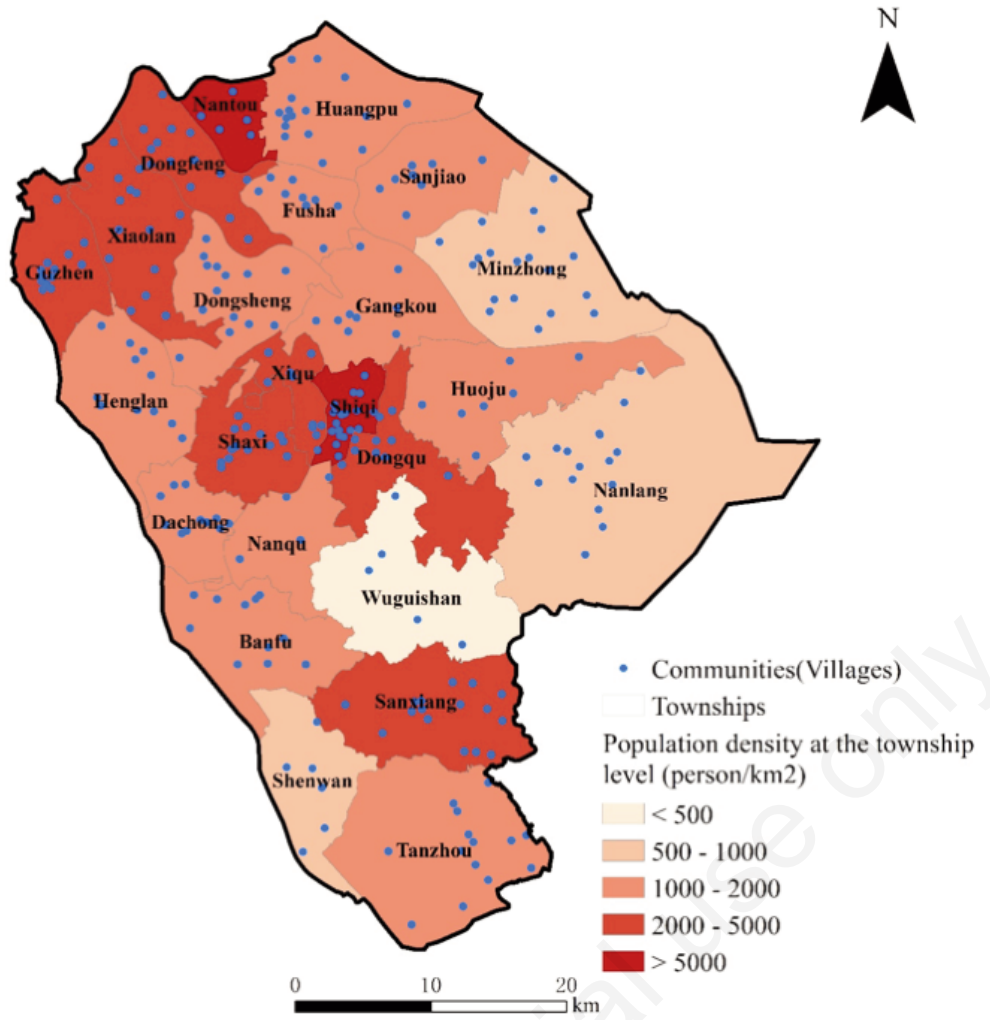

Figure 2. The main urban area of Zhongshan.

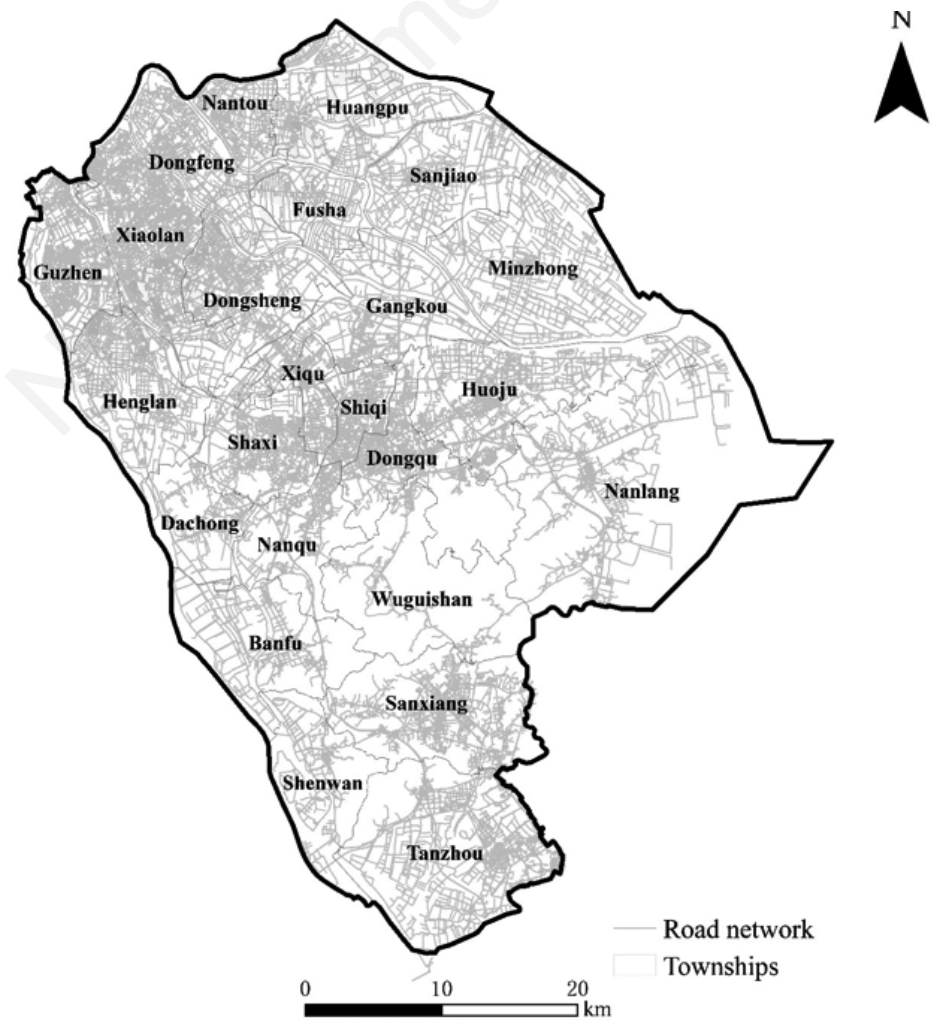

Figure 3. The road network of Zhongshan City. 


\section{Data sources}

Data on healthcare facilities were mainly derived from annual statistical reports on hospitals and primary healthcare facilities provided by the Zhongshan Health Bureau. The primary information incorporates details such as type of institution, address, human resources, finance, service volume, etc. Useful information includes name, address, hospital level, number of doctors and the actual number of beds in each institution. In Zhongshan City, the healthcare system is divided into primary healthcare facilities, which provide services ranging from diagnosis and treatment of common and chronic diseases to rehabilitation and nursing services, with secondary and tertiary-level hospitals carrying out surgery and being responsible for treating more acute and serious situations, such as emergencies and hospitalizations. Different levels of healthcare serve different needs, with the higher levels covering a wider field than the lower ones. However, since primary care visits are not mandatory in China, this paper assumes that the need for different levels of healthcare is the same (Tao et al., 2020). There are 285 primary facilities, 25 secondary hospitals and 4 tertiary hospitals in Zhongshan. Some private and specialized hospitals were excluded because they were only engaged in a small number of for-profit specialty services, such as dentistry, rehabilitation and medical cosmetology. Finally, 285 primary, $15 \mathrm{sec}-$ ondary and 4 tertiary facilities were included in the study.

Demographic data are often used to indicate the demand for healthcare services (Levesque et al., 2013). Most existing studies, including those dealing with healthcare accessibility, have applied population data at the sub-district level for analysis (Bagheri et al., 2005; Yang et al., 2006). This study focuses on resident population data at the village level, the lowest administrative unit in China. The data were acquired from the administrative department for the migrant population in Zhongshan City. As of the end of 2018, Zhongshan had 24 towns (sub-districts and economic development zones) under its jurisdiction, including 127 resident committees and 150 villagers' committees, with a total population density of 1,856 people per $\mathrm{km}^{2}$. The mean population of each town was $137,917(\mathrm{SD}=69732.5)$, the mean area of each town was $74.38 \mathrm{~km}^{2}$ $(\mathrm{SD}=37.37)$, and the mean population of each community and village was $11,949.46(\mathrm{SD}=10,889.2)$. The addresses of all 304 facilities and 277 settlements were collected through the Amap map geocoding API (https://lbs.amap.com/console/show/picker), a mobile map application with the currently highest market share in China, and manually checked for accuracy. The distance and travel time from all demand nodes to healthcare facilities were also obtained through the Amap API open platform (https://lbs.amap.com/getting-started/path). Some studies have shown that this method is more accurate than calculating the spatial distance and travel time through the road network because the latter is less often updated and has omissions, while the set road speed is not always accurate (Wang and $\mathrm{Xu}, 2011$; Song et al., 2013). The estimated travel time was measured between 9 a.m. and 5 p.m. on workdays to avoid traffic congestions during rush hours.

\section{Accessibility measurements}

The 2SFCA method consists of two basic steps. For each supply point $j$, the first looks for all demand nodes $k$ within the search radius $d_{0}$ to compute the supply-demand ratio $R_{j}$ as follows:

$$
R_{j}=\frac{s_{j}}{\underset{k \in\left\{\mathrm{d}_{k j} \leq d_{0}\right\}}{P_{k}}}
$$

For each demand node $i$, the next step looks for all supply nodes $j$ within the search radius $d_{0}$, and sums up the supplydemand ratios $R_{j}$ to obtain the accessibility $A_{i}^{F}$ for point $i$ as follows:

$$
A_{i}^{F}=\sum_{j \in\left\{\mathrm{d}_{i j} \leq d_{0}\right\}} R_{j}=\sum_{j \in\left\{\mathrm{d}_{i j} \leq d_{0}\right\}}\left[\frac{s_{j}}{\sum_{k \in\left\{\mathrm{d}_{k j} \leq d_{0}\right\}} P_{k}}\right]
$$

where $i$ represents the demand nodes; $j$ the supply point; $A_{i}^{F}$ the accessibility of demand nodes $i$ calculated from 2SFCA; $d_{i}^{j}$ the distance between demand point $i$ and supply point $j ; R_{j}$ the ratio of the size of facility at supply point $j$ to the population served within the catchment area $\left(d_{0}\right) ; S_{j}$ the size of supply at supply point $j$; and $P_{k}$ the size of demand at demand nodes $k$. The original 2SFCA technique Radke and Lan (2000) used on-demand nodes (inhabitants) to calculate the supply-to-demand ratios when searching for supply points within the catchment area, but failed to consider situations where one supply point serves multiple demand nodes. To cope with this deficiency, Luo and Qi (2009) developed an improved version. Both approaches assume that all facility supply points are spatially and optimally configured to meet the demand in the system, which is represented by the accessibility score for each demand node multiplied by its demand size and summed to equal the total facility size according to the evaluation results of the existing 2SFCA model family. Although this implies that all facility resources are fully utilized, this assumption is unlikely to be met in the real world. Therefore, Delamater (2013) proposed an important extension, the Modified 2SFCA (M2SFCA), which allows for a suboptimal spatial configuration of facilities, i.e. that there are some facility resources that cannot be allocated to the demand nodes and thus cannot be effectively utilized as compared to the weighted sum of the demand size of accessibility score mentioned earlier. The problem can be summarized as follows: for all the effective measurements in terms of accessibility, 2SFCA and its subsequent improvements lack consideration of availability. While 2SFCA and its current improved forms measure accessibility well, they do not consider the availability of facility resources, but the M2SFCA version does measure availability based on accessibility (Tao and Cheng, 2016), an improvement that significantly contributed to the development and popularity of 2SFCA and even spatial accessibility modelling. However, it still fails to assess hierarchical facility accessibility (Tao et al., 2020). In response to the features in hierarchical systems, several studies have strived to measure the spatial accessibility to hierarchical facilities by using more comprehensive measures such as the 2SFCA (Tao et al., 2015; Hu et al., 2019; Jin et al., 2019; Li et al., 2019). Nonetheless, these models generally only consider discrepancies in the size of the catchment area between different levels of facilities. In view of the shortcomings of these models, Tao et al. (2020) developed the hierarchical 2SFCA (H2SFCA), allowing for different catchment sizes, distance attenuation effects and transportation modes for different levels of facilities, in which both relative and absolute distances can vary.

This study employed the H2SFCA method to measure the accessibility and general accessibility to three levels of healthcare facilities in Zhongshan City, using the following expression:

$$
G A_{i}=\sum_{l} \sum_{j \in\left\{d_{i j} \leq D^{l}\right\}} \frac{s_{j}^{l} f\left(d_{i j}\right) f\left(d_{i j}\right)}{\sum_{k \in\left\{d_{k j} \leq D\right.} P_{k} f\left(d_{i j}\right)}
$$

where $G A_{i}$ is the general spatial accessibility at location $i$ in a hierarchical system; $l$ the level of facilities in the system; $S_{j}^{l}$ the supply 
of facility $j$ at level $l$; and $D^{l}$ the catchment area size of facilities at level $l$. In this study, the Gaussian function was adopted to model the distance-decay effects as suggested by the aforementioned studies:

$$
f\left(d_{i j}\right)=\left\{\begin{array}{c}
\frac{e^{-1 / 2 \times\left(d_{i j} / D^{l}\right)^{2}-} e^{-1 / 2}}{1-e^{-1 / 2}}, d_{i j} \leq D^{l} \\
0, d_{i j}>D^{l}
\end{array}\right.
$$

The proposed H2SFCA assumes that facilities at various levels have different service areas (i.e. catchment areas) in a hierarchical system. According to the setting of related research (Jin et al., 2019), we assumed that walking was the major way to reach primary healthcare facilities, while driving was the most common when travelling to secondary and tertiary-level hospitals. The catchment area sizes $D^{l}$ were set at 30,50 and $70 \mathrm{~min}$ for facilities at three levels. Based on the above data and settings, the spatial accessibility, as well as the general accessibility, at each demand location (i.e. villages \& communities) at each level of healthcare facilities, could be estimated. In addition, since the accessibility values measured by H2SFCA do not directly reflect travel distance or time, we compiled the results based on the method estimating travel impedance to the nearest provider for different travel times or distance ranges. As an important supplement to the H2SFCA measurement results, they counted the accessibility of each demand node using different distances or time ranges. Data crawling and cleaning were completed through $\mathrm{R}$ studio 4.0.2, and all accessibility calculations and charting were completed through ArcGIS 10.5 (ESRI, Redlands, CA, USA).

\section{Results}

\section{Baseline distribution of healthcare facilities}

The distribution of healthcare facilities was found to vary considerably. Figure 4 shows the spatial distribution of 304 public medical facilities in Zhongshan and the corresponding population density in each township. Three of the four tertiary-level hospitals are located in the traditional central urban areas (Xiqu, Dongqu and Shiqi). Another such hospital is located in the north-western region with a relatively

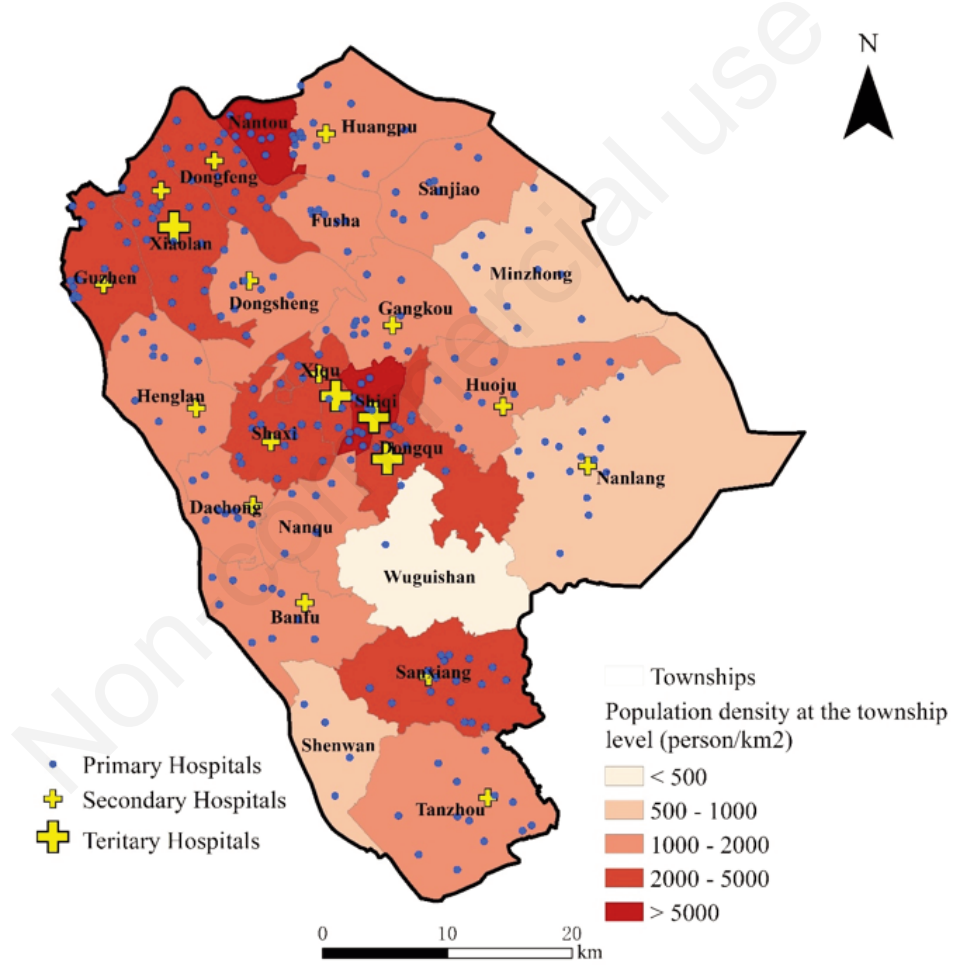

Figure 4. the spatial distribution of public medical facilities in Zhongshan City in comparison with the corresponding population density in each township.

Table 2. Applying population weights to accessibility.

\begin{tabular}{lccc} 
Level & Primary & Secondary & Tertiary \\
Population-weighted average accessibility* & 6.87 & 10.42 & 19.09 \\
Total supply (number of physicians) & 1833 & 2143 & 2643 \\
\hline Number of facilities & 285 & 15 & 4 \\
Standard deviation (multiplying by 10,000) & 5.04 & 3.66 & 5.08 \\
\hline Coefficient of variation (CV) & 0.86 & 0.65 & 0.78 \\
\hline
\end{tabular}

*Equal to the accessibility of communities (villages) multiplied by their population, summed up and divided by the number of settlements. 
dense population, but none in the remaining areas. Overall, the distribution of the 15 secondary-level hospitals is balanced but not all towns have one, not even Nantou, which is a densely populated area. The spatial distribution of primary medical institutions, on the other hand, is roughly similar to that of the population.

\section{Spatial healthcare accessibility}

We measured the average accessibility to each level of healthcare facility by applying population weights to the accessibility as shown in Table 2. Given that different levels of facilities had the same population and similar needs of healthcare, we could compare the accessibility to the three levels mentioned. The results indicate that although primary healthcare facilities outnumbered secondary and tertiary facilities, the average accessibility presented the opposite tendency. Specifically, the tertiary-level hospitals had the highest weighted average accessibility followed by the secondary facilities whereas primary healthcare facilities had the lowest. By definition, accessibility depends on the number of physicians and the travel time between home and facility for residents. Moreover, it was found that tertiary hospitals had the highest number of physicians, with an average of 661 physicians per facility, which was 4.62 times the figure of the secondary-level healthcare facilities (143) and 103 times that of the primary facilities (6.43).

Figure 5 shows the spatial accessibility with respect to hospital level. The distribution of accessibility to tertiary-level hospitals demonstrated clear clustering, i.e. the central city and its surrounding areas had the highest accessibility, while the north-eastern towns Huangpu and Minzhong, as well as the southern Sanxiang and Tanzhou had the lowest. There are four tertiary-level hospitals in Zhongshan, three of which in the central city, providing residents with better accessibility, but few located outside the city centre. Although a tertiary hospital has been built in the more economically developed town Xiaolan, this is not sufficient, and the uneven distribution of quality healthcare resources continues to be a problem.
Unlike tertiary-level hospitals that have strong central accessibility, the accessibility of secondary-level hospitals is not well balanced with better access in the north-western and south-eastern parts of the city represented by the towns Guzhen, Xiaolan, Sanxiang and Tanzhou and less in the central and eastern regions. This phenomenon may be a result of the geographical distribution of secondary hospitals because most of these are located in the areas mentioned above, further strengthening their accessibility. There are no secondary hospitals in Nanqu, Shiqi, Dongqu, Minzhong and Sanjiao towns, which are situated at the central and eastern regions. Coupled with the high population density in the centre, these areas thus have low accessibility to secondary hospitals.

The spatial distribution of primary healthcare facilities is most even among the three levels of healthcare facilities, but the spatial accessibility was found to be generally low. High accessibility exists in some areas, such as Nantou, Fusha, Sanjiao, Shenwan and Sanxiang towns, but this is not equivalent to the adequate supply of healthcare services provided by the primary healthcare facilities. Part of the reason may be the relatively low population density in some of these areas. Also, while the accessibility in western and central areas of Zhongshan was found to be low, it was not better in the central urban area with its high population density.

Table 3 shows the accessibility results based on minimum distance and travel time, and it was found that the minimum distance from the demand nodes (villages and communities) to the primary hospital is generally $2 \mathrm{~km}$ or less $(81.2 \%)$, while that to the secondary-level hospital is mainly more than $2 \mathrm{~km}(87.4 \%)$ and the distance to the tertiary hospital often more than $5 \mathrm{~km}(78.0 \%)$. In addition, the minimum time from the demand nodes to the primary hospital is mostly 20 minutes or less $(76.5 \%)$, the minimum time to the secondary hospital in most cases $10-30$ minutes $(70.8 \%)$, while the time to the tertiary-level hospital is most commonly more than 20 minutes $(77.6 \%)$.
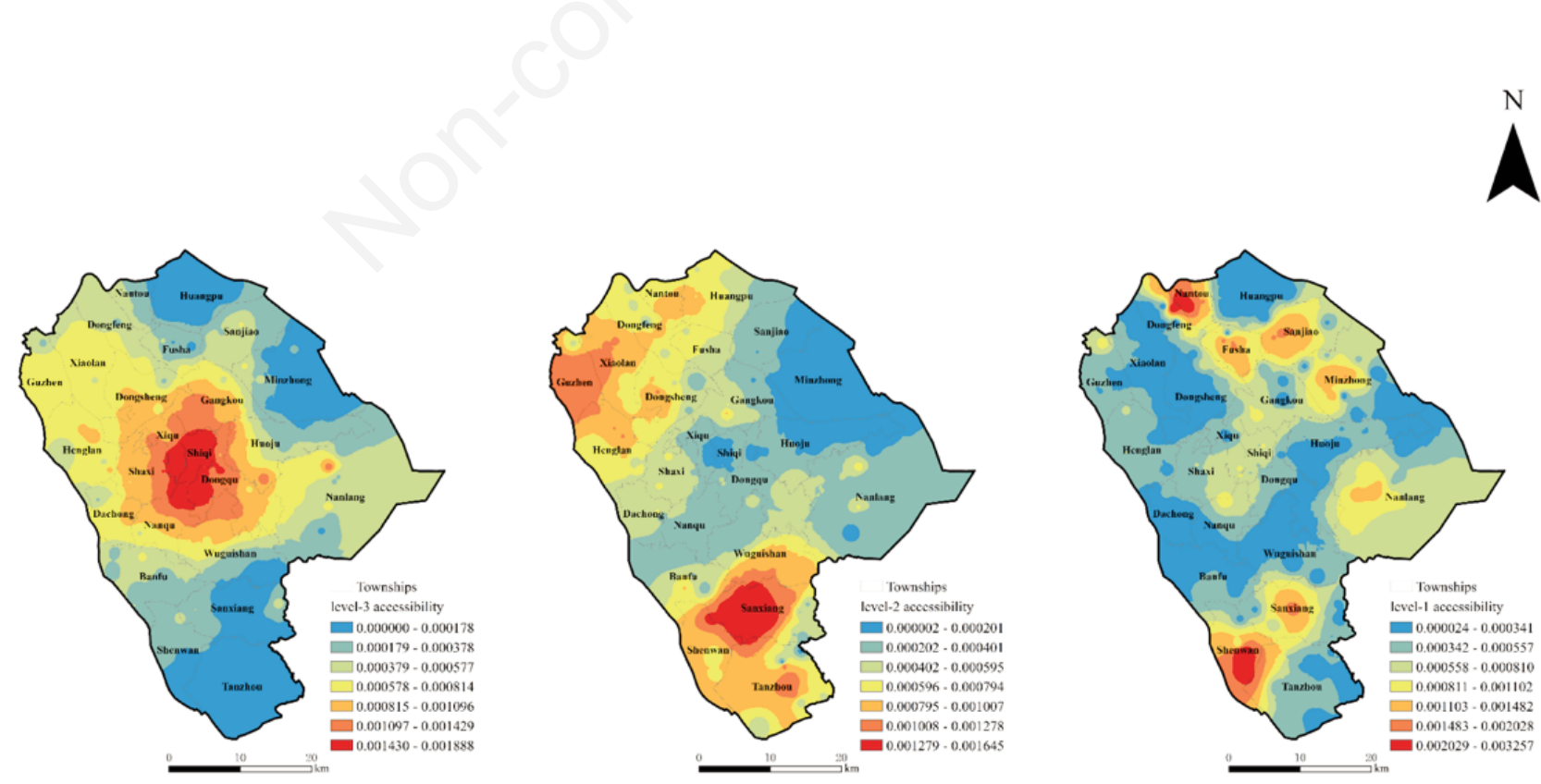

Figure 5. The spatial accessibility with respect to hospital level in Zhongshan City. 


\section{General healthcare accessibility}

The general accessibility to healthcare in Zhongshan was attained by summing the accessibility to the three levels of hospitals (Eq. 3). The results indicate that the accessibility is distributed in spatial clusters, with a strong dependence on distance to hospi- tals and decaying as the distance to hospitals shortens. As shown in Figure 6, the accessibility to healthcare services in Zhongshan is substantially unevenly distributed, with the highest accessibility concentrated in the central city, Nantou Town in northern Zhongshan and the towns Shenwan and Sanxiang in the South. It was noticeable that these areas are among the most densely popu-

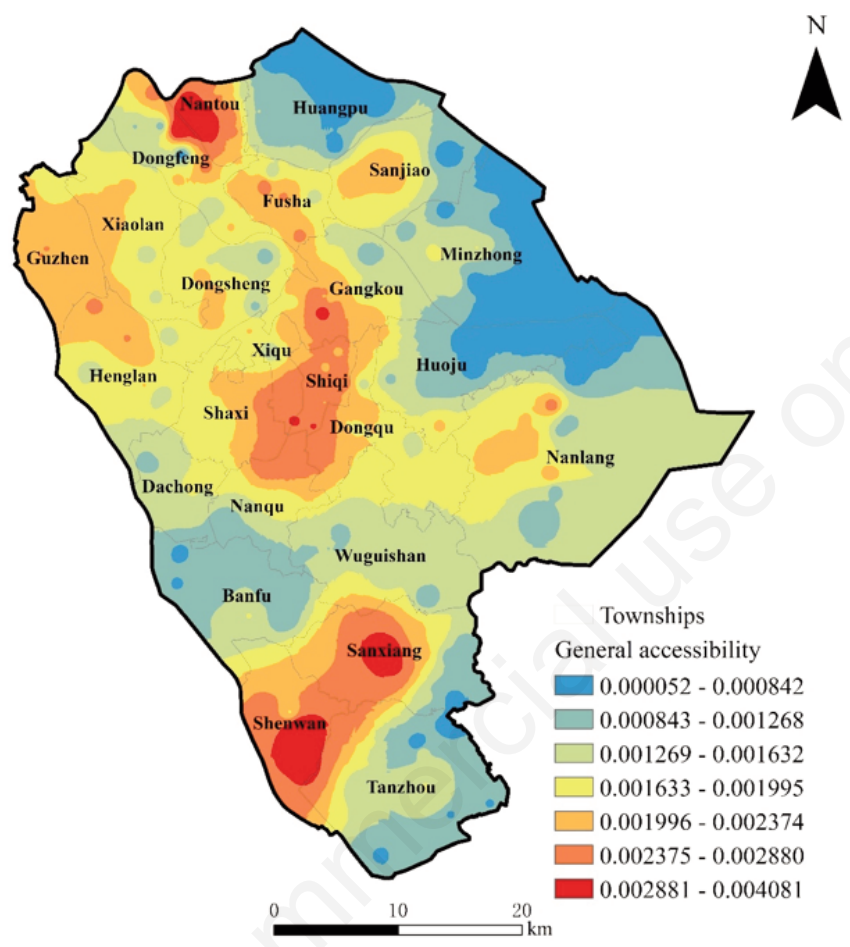

Figure 6. The accessibility to healthcare services in Zhongshan City.

Table 3. Applying accessibility results based on minimum distance and travel time.

\begin{tabular}{|c|c|c|c|c|c|c|c|c|}
\hline & Primary hospital & & & Secondary hospit & & & rtiary hospital & \\
\hline No. & $\begin{array}{l}\text { Accessibility } \\
\text { accessibility } \\
\text { rate* } \\
(\%)\end{array}$ & $\begin{array}{c}\text { Cumulative } \\
\text { rate } \\
(\%)\end{array}$ & $\begin{array}{l}\text { No. } \\
\text { (\%) }\end{array}$ & $\begin{array}{l}\text { Accessibility } \\
\text { accessibility } \\
\text { rate } \\
(\%)\end{array}$ & $\begin{array}{l}\text { Cumulative } \\
\text { rate }\end{array}$ & No. & $\begin{array}{l}\text { Accessibility } \\
\text { accessibility } \\
\text { rate } \\
(\%)\end{array}$ & $\begin{array}{l}\text { Cumulative } \\
\text { rate } \\
(\%)\end{array}$ \\
\hline
\end{tabular}

\section{Minimum}

distance $^{\circ}(\mathrm{km})$

\begin{tabular}{cccccccccc}
$0-1$ & 100 & 36.10 & 36.10 & 8 & 2.89 & 2.89 & 2 & 0.72 & 0.72 \\
$1-2$ & 125 & 45.13 & 81.23 & 27 & 9.75 & 12.64 & 8 & 2.89 & 3.61 \\
$2-5$ & 50 & 18.05 & 99.28 & 79 & 28.52 & 41.16 & 39 & 14.08 & 17.69 \\
$5-10$ & 1 & 0.36 & 99.64 & 107 & 38.63 & 79.79 & 55 & 19.86 & 37.55 \\
$>10$ & 1 & 0.36 & 100.00 & 56 & 20.21 & 100.00 & 173 & 62.45 & 100.00 \\
Minimum & & & & & & & & & \\
ime (min) & & & & & & & & & \\
$0-10$ & 18 & 28.16 & 28.16 & 52 & 18.77 & 18.77 & 9 & 3.25 & 3.25 \\
$10-20$ & 134 & 48.38 & 76.54 & 102 & 36.82 & 55.59 & 44 & 15.88 & 19.13 \\
$20-30$ & 48 & 17.33 & 93.87 & 94 & 33.94 & 89.53 & 54 & 19.49 & 38.62 \\
$>30$ & 17 & 6.13 & 100.00 & 29 & 10.47 & 100.00 & 170 & 61.38 & 100.00 \\
\hline
\end{tabular}

*Obtained in two steps: the first step is to identify n, the number of demand nodes (communities or villages), which have the minimum distance or spend the minimum time to primary, secondary and tertiary hospitals; the second step is to calculate the proportion of $n$ among all demand nodes; ${ }^{\circ}$ walking assumed to be the major way to reach primary healthcare facilities and driving the major way to reach secondary and tertiary hospitals. The minimum time and minimum distance were obtained on this basis through the AMap API. 
lated ones, particularly the central city and Nantou Town, indicating that Zhongshan needs to deliver a widening access to healthcare. Furthermore, since H2SFCA considered healthcare needs of neighbourhoods, service capacity of facilities and distance of transportation, the uneven distribution of accessibility was indicative of a highly unbalanced demand and supply. We found that the accessibility in the north-western region with high density was generally lower than that in regions with high accessibility, a situation that becomes manifest when the distribution of healthcare resources is more centralized than the population. Thus, there is still a mismatch between population distribution and medical services.

\section{Discussion}

Our study supplements the existing literature in measuring the accessibility to hierarchical healthcare facilities in mid-sized cities in China under the context of hierarchical healthcare delivery system reform. The H2SFCA method, used to measure the spatial accessibility to various levels of healthcare facilities in Zhongshan, showed that the spatial distribution of accessibility to hierarchical healthcare facilities was uneven, with high-accessibility clusters in the centre, parts of the Northwest and parts of the South. Local governments should rationally allocate healthcare resources and improve the capacity of primary services to ameliorate the overall accessibility to healthcare services.

Notably, most villages (communities) have relatively high travel costs to tertiary-level hospitals, with more than $70 \%$ of them situated at distances greater than $5 \mathrm{~km}$, which generally takes longer than 20 minutes to cover. The H2SFCA calculations also showed that the concentration of tertiary hospitals in the central city area leads to significant differences in general accessibility. Similar to the results of space accessibility measurements in Shenzhen (Cheng et al., 2016; Tao et al., 2020), tertiary hospitals have a higher accessibility value in Zhongshan compared to secondary and primary hospitals and therefore has a greater impact on general accessibility. Tertiary-level hospitals constitute the top of China's hierarchical healthcare system, bringing together the best healthcare resources ranging from highly educated doctors to large numbers of beds and state-of-the-art equipment. Besides, because their wider purview, including comprehensive primary care and research, is seen by the general public as providing higher-quality services (Eggleston et al., 2008; Qingyue et al., 2019), which increases the demand for their services. Therefore, the construction of new large tertiary hospitals in peripheral areas could effectively reduce the inequity of healthcare services (Tao et al., 2018). To meet the healthcare needs of residents, the Government should consider the potential healthcare needs of the residents and establish several tertiary hospitals or branches in low-accessibility areas, such as the towns Minzhong, Huangpu and Banfu.

Primary healthcare is widely perceived to be the backbone of a national healthcare system (Starfield, 1994; Starfield et al., 2005; Li et al., 2017), with accessibility considered a fundamental right and an important facilitator of overall population health (Dewulf et al., 2013). Although $81.2 \%$ of demand nodes means less than $2 \mathrm{~km}$ travel to the nearest primary hospital, H2SFCA shows lower accessibility to primary healthcare facilities, suggesting that the supply of medical resources in primary healthcare is far from adequate relative to its functional orientation. Although the Zhongshan City Government is planning for 205 primary healthcare facilities, these will be insufficiently equipped as they generally will lack qualified physicians. In addition, similar to other studies (McGrail, 2012; Dewulf et al., 2013; Wang et al., 2018), a large disparity emerges in primary healthcare resources in Zhongshan, since there is a large difference in accessibility between the central city area and other areas, especially in the relatively densely populated north-eastern areas (Xiaolan, Dongsheng and Dongfeng towns). This indicates that although these areas are urbanized, public resources such as healthcare fail to keep pace with the demand for primary health care. As it has been shown that the capacity of primary healthcare facilities is unlikely to be enhanced quickly, the Government should increase funding for primary healthcare in these areas, take advantage of better spatial distribution of community health centres, transfer quality healthcare resources from higher-level hospitals to primary healthcare facilities and provide easy access to healthcare services for the residents (Hu et al., 2019). In addition, the government should produce a rational layout of secondary care facilities with measures incorporating increasing health funding for townships that do not have them. This means upgrading a number of qualified primary facilities to secondary hospitals to meet regional demand for complex disease diagnosis and treatment.

Zhongshan is not an exception to the challenge of uneven spatial accessibility to urban healthcare resources. Tao et al. (2020) measured the spatial accessibility to hierarchical healthcare facilities in Shenzhen through 2SFCA and concluded that the overall spatial accessibility to hierarchical healthcare facilities in Shenzhen is unevenly distributed. Zhao et al. (2020) used E2SFCA, another version of the 2SFCA methodology; to measure the accessibility to tertiary and secondary hospitals in Beijing and found that compared with communities in the central city area, suburban communities have less access to medical services, an unequal medical service space that seems to be intensifying. Unlike the frequently studied metropolitan cities, parts of the distant urban areas of Zhongshan show a high degree of accessibility, suggesting that the development of healthcare resources in Zhongshan may be more balanced than those in some other large cities. However, the accessibility to healthcare facilities at all levels is low, but at varying degrees. In 2015, the Chinese Government implemented referral reform in its hierarchical medical system by adjusting the reimbursement rules of medical insurance to guide patients' hospital preferences. This reform has affected the equity and spatial accessibility to healthcare resources in different regions. The study by Lu et al. (2019) showed that the referral reform improved total accessibility to public hospitals in Beijing. In light of the findings of this study in Zhongshan, we believe that there is still a long way to go before the accessibility to healthcare are clearly strengthened. To achieve improved access to medical resources for residents and promote equitable use of health services, the Government should incorporate spatial allocation of resources into health resource planning, rationally allocate medical resources at all levels and improve the hierarchical diagnosis and treatment and medical insurance systems.

There are several limitations noted in this study, which can be improved in the future. Firstly, the use of population figures for each neighbourhood as an approximate proxy for need does not consider the specific healthcare needs of different types of patients, such as geriatric, dental, mental as well as situations such as emergencies, etc. This could be ameliorated by acquiring standardized administrative electronic health records (EHRs) data and census data. Secondly, limited by data controls, the administrative boundary vector data for each administrative village (community) are not available, which may exert influence on the visualization of spatial accessibility, but the Kriging spatial interpolation approach (Oliver and Webster, 1990) allows for a good visual representation of the 
spatial distribution of accessibility. Finally, due to limitations of the model per se, other features of the hierarchical facilities, such as specialized functions of each level and economies of scale at each level, are still excluded from the approach (Tao et al., 2020).

\section{Conclusions}

China is currently implementing hierarchical diagnosis and treatment reform, but there are few studies focusing on accessibility to healthcare in medium-sized cities. The study carried out found that the spatial distribution of accessibility to hierarchical healthcare facilities in Zhongshan remains uneven, with clusters of high accessibility areas in the centre and parts of areas in the Northwest and South. It is urgent to develop new models and employ new technologies to study the allocation of healthcare resources in all types of cities. Local Governments should rationally allocate healthcare resources and improve the capacity of primary services to enhance overall accessibility. Equal access to healthcare services would also be beneficial to all in the context of curbing the COVID-19 pandemic.

\section{References}

Banister D, 1996. Transport and urban development. E \& FN Spon, an imprint of Chapman \& Hall, London, UK, pp. 28-30.

Bauer J, Groneberg DA, Maier W, Manek R, Louwen F, Brüggmann D, 2017. Accessibility of general and specialized obstetric care providers in Germany and England: an analysis of location and neonatal outcome. Int J Health Geogr 16:44.

Bagheri N, Benwell GL, Holt A, 2005. Measuring spatial accessibility to primary health care. Paper presented at the 17 th Annual Colloquium of the Spatial Information Research Centre, University of Otago, New Zealand.

Central Committee of the Communist Party of China, 2020. Proposals for formulating the 14th Five-Year Plan (2021-2025) for National Economic and Social Development and the LongRange Objectives Through the Year 2035. Available from: http://www.gov.cn/zhengce/2020-11/03/content_5556991.htm Accessed: 24 April 2021.

Cheng G, Zeng X, Duan L, Lu X, Sun H, Jiang T, 2016. Spatial difference analysis for accessibility to high level hospitals based on travel time in Shenzhen, China. Habitat Int 53:48594.

Delamater PL, 2013. Spatial accessibility in suboptimally configured health care systems: a modified two-step floating catchment area (M2SFCA) metric. Health Place 24:30-43.

Dewulf B, Neutens T, De Weerdt Y, Van de Weghe N. 2013. Accessibility to primary health care in Belgium: an evaluation of policies awarding financial assistance in shortage areas. BMC Family Pract 14:122.

Eggleston K, Ling L, Qingyue M, Lindelow M, Wagstaff A, 2008. Health service delivery in China: a literature review. Health Econ 17:149-65.

Guagliardo MF, 2004. Spatial accessibility of primary care: concepts, methods and challenges. Int J Health Geograph 3:1-13.

Gu X, Zhang L, Tao S, Xie B, 2019. Spatial accessibility to healthcare services in metropolitan suburbs: the case of Qingpu, Shanghai. Int J Environ Res Public Health 16:225.

Health Bureau of Zhongshan , 2018. A snapshot of the development of health resources and medical services in Zhongshan in
2018. Available from: http://wjj.zs.gov.cn/xxml/tjgh/content/ post_62028.html Accessed: 24 April 2021.

$\mathrm{Hu}$ W, Li L, Su M, 2019. Spatial inequity of multi-level healthcare services in a rapid expanding immigrant city of China: a case study of Shenzhen. Int J Environ Res Public Health 16:3441.

Jay P, David S. 2016. Geographic distribution of hospital beds throughout China: a county-level econometric analysis. Int $\mathrm{J}$ Equity Health 15:1-8.

Jin M, Liu L, Tong D, Gong Y, Liu Y, 2019. Evaluating the spatial accessibility and distribution balance of multi-level medical service facilities. Int J Environ Res Public Health 16:7.

Li L, Fu H, 2017. China's health care system reform: progress and prospects. Int J Health Plan Manage 32:240-53.

Liu GG, Vortherms SA, Hong X, 2017. China's health reform update. Annu Rev Public Health 38:431-48.

Li X, Lu J, Hu S, Cheng K, De Maeseneer J, Meng Q, 2017. The primary health-care system in China. Lancet 390:2584-94.

Luo W, Qi Y, 2009. An enhanced two-step floating catchment area (E2SFCA) method for measuring spatial accessibility to primary care physicians. Health Place 15:1100-107.

Lu C, Zhang Z, Lan X, 2019. Impact of China's referral reform on the equity and spatial accessibility of healthcare resources: a case study of Beijing. Soc Sci Med 235:112386.

Levesque JF, Harris MF, Russell G, 2013. Patient-centred access to health care: conceptualising access at the interface of health systems and populations. Int J Equity Health 12:18.

Li L, Du Q, Ren F, Ma X, 2019. Assessing spatial accessibility to hierarchical urban parks by multi-types of travel distance in Shenzhen, China. Int J Environ Res Public Health 16:1039.

McGrail MR, 2012. Spatial accessibility of primary health care utilising the two step floating catchment area method: an assessment of recent improvements. Int J Health Geogr 11:50.

National Health Commission, 2019. China health statistics yearbook 2019. Peking Union Medical College Press, Beijing, China.

National Health Commission, 2020. China Health Statistics Yearbook. Peking Union Medical College Press, Beijing, China.

Oliver MA, Webster R, 1990. Kriging: a method of interpolation for geographical information systems. Int J Geograph Inf Syst 4:313-32.

Pan J, Liu H, Wang X, Xie H, Delamater PL, 2015. Assessing the spatial accessibility of hospital care in Sichuan Province, China. Geospat Health 10:384.

Qingyue M, Daoxin Y, Anne M, Kamran A, 2019. China's encouraging commitment to health. BMJ 365:14178.

Radke J, Lan M, 2000. Spatial decompositions, modeling and mapping service regions to predict access to social programs. Geogr Inf Sci 6:105-12.

Shan L $\square$ Qunhong W, Chaojie L, Ye L, Yu C, Zi L, Yanhua H, Libo L, Ning N, Ding D, Qingxia P, Liyuan H, 2017. Perceived challenges to achieving universal health coverage: a cross-sectional survey of social health insurance managers/administrators in China. BMJ Open 7:e014425.

Song S, Yuan B, Zhang L, Cheng G, Zhu W, Hou Z, 2018. Increased Inequalities in Health Resource and Access to Health Care in Rural China. Int J Environ Res Public Health 16:49.

Song P, Zhu Y, Mao X, Li Q, An L, 2013. Assessing spatial accessibility to maternity units in Shenzhen, China. PLoS One 8:e70227.

Starfield B, 1994. Is primary care essential? Lancet 344:1129-33.

Starfield B, Shi L, Macinko J, 2005. Contribution of primary care to health systems and health. Milbank Q 83:457-502. 
Tediosi F, Finch A, Procacci C, Marten R, Missoni E, 2016. BRICS countries and the global movement for universal health coverage. Health Policy Plan 31:717-28.

Tao Z, Cheng Y, Liu J, 2020. Hierarchical two-step floating catchment area (2SFCA) method: measuring the spatial accessibility to hierarchical healthcare facilities in Shenzhen, China. Int J Equity Health 19:164.

Tao Z, Cheng Y, 2016. Research progress of the two-step floating catchment area method and extensions. Progr Geogr 35:58999.

Tao Z, Cheng Y, Dai T, Li X, 2015. Spatial optimization of residential care facility locations in 2020 in Beijing:maximum equity in accessibility. Progr Geogr 34:1609-616.

Tao Z, Yao Z, Kong H, Duan F, Li G. 2018. Spatial accessibility to healthcare services in Shenzhen, China: improving the multimodal two-step floating catchment area method by estimating travel time via online map APIs. BMC Health Serv Res 18:345.

World Health Organization, 2005. Sustainable health financing, universal coverage and social health insurance. World Health Assemb Resol 58:139-40.

Wang F, Xu Y, 2011. Estimating O-D travel time matrix by Google Maps API: implementation, advantages, and implications. Annals of GIS, 17(4), 199-209. doi:10.1080/19475683.
2011.625977

Wang X, Yang H, Duan Z, Pan J, 2018. Spatial accessibility of primary health care in China: a case study in Sichuan Province. Soc Sci Med 209:14-24.

Yip W, Fu H, Chen AT, Zhai T, Jian W, Xu R, Chen W, 2019. 10 years of health-care reform in China: progress and gaps in Universal Health Coverage. Lancet 394:1192-204.

Yue Z, Qian W, Tian J, Jian W. 2018. Equity and efficiency of primary health care resource allocation in mainland China. Int $\mathrm{J}$ Equity Health 17:1-12.

Yang DH, Goerge R, Mullner R, 2006. Comparing GIS-based methods of measuring spatial accessibility to health services. J Med Syst 30:23-32.

Zhai K, Hou S, 2020. Green framework, implication and promotion scheme of high-quality development of urban-rural integration in China during the 14th Five-Year Plan Period. Reform 11:53-68.

Zhongshan Bureau of Statistics, 2019. Zhongshan statistical yearbook 2019. Available from: http://stats.zs.gov.cn/zwgk/ tjxx/tjnj/content/post 1342223.html Accessed: 24 April 2021.

Zhao P, Li S, Liu D, 2020. Unequable spatial accessibility to hospitals in developing megacities: new evidence from Beijing. Health Place 65:102406. 